\title{
EB1-Microtubule Interactions in Xenopus Egg Extracts: Role of EB1 in Microtubule Stabilization and Mechanisms of Targeting to Microtubules
}

\author{
Jennifer S. Tirnauer, ${ }^{*+}$ Sonia Grego, ${ }^{\ddagger}$ E.D. Salmon, ${ }^{\ddagger}$ and \\ Timothy J. Mitchison*
}

\begin{abstract}
*Department of Cell Biology, Harvard Medical School, Boston, Massachusetts 02115; and łDepartment of Biology, University of North Carolina at Chapel Hill, Chapel Hill, North Carolina 27599
\end{abstract}

Submitted April 17, 2002; Revised July 9, 2002; Accepted July 17, 2002

Monitoring Editor: J. Richard McIntosh

\begin{abstract}
EB1 targets to polymerizing microtubule ends, where it is favorably positioned to regulate microtubule polymerization and confer molecular recognition of the microtubule end. In this study, we focus on two aspects of the EB1-microtubule interaction: regulation of microtubule dynamics by EB1 and the mechanism of EB1 association with microtubules. Immunodepletion of EB1 from cytostatic factor-arrested M-phase Xenopus egg extracts dramatically reduced microtubule length; this was complemented by readdition of EB1. By time-lapse microscopy, EB1 increased the frequency of microtubule rescues and decreased catastrophes, resulting in increased polymerization and decreased depolymerization and pausing. Imaging of EB1 fluorescence revealed a novel structure: filamentous extensions on microtubule plus ends that appeared during microtubule pauses; loss of these extensions correlated with the abrupt onset of polymerization. Fluorescent EB1 localized to comets at the polymerizing plus ends of microtubules in cytostatic factor extracts and uniformly along the lengths of microtubules in interphase extracts. The temporal decay of EB1 fluorescence from polymerizing microtubule plus ends predicted a dissociation half-life of seconds. Fluorescence recovery after photobleaching also revealed dissociation and rebinding of EB1 to the microtubule wall with a similar half-life. EB1 targeting to microtubules is thus described by a combination of higher affinity binding to polymerizing ends and lower affinity binding along the wall, with continuous dissociation. The latter is likely to be attenuated in interphase. The highly conserved effect of EB1 on microtubule dynamics suggests it belongs to a core set of regulatory factors conserved in higher organisms, and the complex pattern of EB1 targeting to microtubules could be exploited by the cell for coordinating microtubule behaviors.
\end{abstract}

\section{INTRODUCTION}

Multiple diverse proteins regulate the polymerization dynamics of microtubules (reviewed in Cassimeris and Spittle, 2001). Together, these regulatory factors enhance the intrinsic dynamic instability of microtubules in vivo over that in vitro and in mitosis compared with interphase. Studies have suggested that some factors also produce regional effects on microtubules, as seen in the leading edges of polarized cells (Wadsworth, 1999; Akhmanova et al., 2001; Palazzo et al., 2001) and at kinetochore vs. nonkinetochore microtubules

Article published online ahead of print. Mol. Biol. Cell 10.1091/ mbc.E02-04-0210. Article and publication date are at www.molbiolcell.org/cgi/doi/10.1091/mbc.E02-04-0210.

+ Corresponding author. E-mail address: jennifer_tirnauer@hms.

harvard.edu.
(Zhai et al., 1995). Although effects on microtubule stability have been demonstrated for many proteins, Kinoshita et al. (2001) recently reported that a minimal pair of microtubule regulatory proteins, XMAP-215 and XKCM1, was sufficient to achieve near physiological rates of microtubule dynamic instability in vitro. These results lead the authors to conclude that dynamic instability in Xenopus egg extracts is derived solely from the action of these two factors (Kinoshita et al., 2001). However, it is likely that cells use other microtubule regulatory proteins besides XMAP-215 and XKCM1 to build on (or diverge from) this minimal system to meet the specialized needs of cell division and differentiation. For example, regional changes in microtubule dynamics during interphase contribute to reorientation of the microtubule organizing center in activated lymphocytes, whereas increases in catastrophe frequency during mitosis allow more efficient searching of space for capture of attachment sites on 
kinetochores (reviewed in Desai and Mitchison, 1997). Local action of other microtubule regulatory proteins is likely to contribute to these local changes in microtubule stability. Understanding how the cell uses its available microtubule regulators will require a combination of detailed mechanistic studies and in vivo observations.

EB1 family proteins, conserved from humans to yeasts and plants, are small $(\sim 35-\mathrm{kDa})$ proteins that specifically recognize the polymerizing plus ends of microtubules (Tirnauer and Bierer, 2000; Schuyler and Pellman, 2001a). In this location, they are positioned to modify the structure of the protofilament ends and thus to regulate microtubule polymerization dynamics, as well as to "flag" the plus end as a recognition site for other subcellular structures (Schroer, 2001). The budding yeast EB1 homolog Bim1p serves both roles: Bim1p promotes microtubule dynamicity by increasing transitions and reducing pauses (Tirnauer et al., 1999), and it spatially marks microtubule plus ends for linkage to the cell cortex, mediated by its binding to the cortical protein Kar9p (Lee et al., 1999; Tirnauer et al., 1999; Korinek et al., 2000; Miller et al., 2000). In the fission yeast Schizosaccharomyces pombe, disruption of the EB1 homologue Mal3 causes chromosome loss, implicating similar roles for EB1 on kinetochore microtubules (Beinhauer et al., 1997). Further support for the role of EB1 at the kinetochore comes from recent studies showing microtubule polymerization-specific targeting of EB1 to kinetochores (Tirnauer et al., 2002), and loss of microtubule-kinetochore interactions in cells lacking the EB1 binding partner adenomatous polyposis coli (APC), (Fodde et al., 2001; Kaplan et al., 2001).

Details of EB1 function at microtubule ends in higher eukaryotes, including whether EB1 promotes microtubule stability, end-on attachment, or both, are unknown. Overexpression of GFP-EB1 in tissue culture cells produces long microtubules, but the specific mechanism has not been described (Bu and $\mathrm{Su}, 2001)$. EB1 has been reported to bind microtubules in vitro (Berrueta et al., 1998), but it fails to induce microtubule polymerization when combined with purified tubulin (Nakamura et al., 2001). Like other microtubule plus end tracking proteins, EB1 forms comet-shaped streaks on polymerizing microtubule ends (Mimori-Kiyosue et al., 2000). Here, we ask how EB1 promotes microtubule stability during meiosis and interphase, and what determines the binding pattern of EB1 to microtubules. We performed these experiments in cytoplasmic extracts prepared from Xenopus laevis eggs, because these extracts can be stably arrested in interphase and $\mathrm{M}$ phase, and they are convenient for protein addition and depletion and high-resolution imaging. These analyses are essential to forming a comprehensive picture of how EB1 integrates with other microtubule dynamics regulators and polarity markers at various sites within the cell.

\section{MATERIALS AND METHODS}

\section{Xenopus Egg Extract Preparation, Immunodepletion, and Western Blotting}

Crude extracts were prepared from $X$. laevis eggs as described previously (Desai et al., 1999a). Cytostatic factor (CSF)-arrested extracts were made and used without addition of nondegradable cyclin or phosphatase inhibitors. Interphase-arrested extracts were made from eggs cycled into interphase with the calcium ionophore
A23187 (Sigma-Aldrich, St. Louis, MO) at $1 \mu \mathrm{g} / \mathrm{ml}$ for $5 \mathrm{~min}$, with the crushing spin carried out at $4^{\circ} \mathrm{C}$; cyclohexamide (Sigma-Aldrich) was then added to the extracts to a final concentration of $100 \mu \mathrm{g} / \mathrm{ml}$ before freezing to prevent synthesis of cyclin B and its activation of the mitotic cdc2 kinase. All extracts were aliquoted (10-100 $\mu \mathrm{l})$ in polymerase chain reaction tubes and flash frozen in liquid nitrogen before use. Cell cycle state of the extracts was confirmed before and after thawing by visual inspection of the morphology of Xenopus sperm nuclei DNA incubated in the extracts.

Immunodepletions were performed on fresh interphase- and CSF-arrested extracts by using a monoclonal antibody (mAb) to EB1 (GD10; generous gift of Marilee Burrell, Oncogene Science, Cambridge, MA). Incubations in the ratio of $150 \mu \mathrm{l}$ of extract to $25 \mu \mathrm{l}$ of protein A-agarose beads (precoated in $1 \mathrm{mg} / \mathrm{ml}$ fraction $\mathrm{V}$ bovine serum albumin [BSA]; Sigma-Aldrich) to $24 \mu \mathrm{g}$ of antibody (GD10 or mouse IgG) were done in the cold for $2 \mathrm{~h}$ with continuous rotation. Each set of immunodepletions was confirmed by Western blotting. Liquid chromatography/mass spectrometry (LC/MS) of the $35-\mathrm{kDa}$ protein eluted from the beads showed homology to human EB1 but not EBF3; and the antibody to human EB1 recognized a single band that matched the mobility of the depleted protein but not Xenopus EBF3 (our unpublished data), suggesting that only the EB1 homologue was depleted. LC/MS was used to identify other proteins codepleted with EB1, and these were confirmed with Western blotting.

Western blotting was done to assess the degree of immunodepletion and to calculate the approximate stoichiometry of EB1 in Xenopus egg extracts. Xenopus egg extracts and bacterially purified human EB1 protein (see below) were boiled in sample buffer, separated on $10 \%$ SDS-PAGE gels, transferred to polyvinylidene difluoride (PVDF; Millipore, Bedford, MA), blocked with 1-2\% BSA in Tris-buffered saline/Tween 20, and probed for EB1 by using the GD10 antibody diluted 1:5000 in Tris-buffered saline/Tween 20. Antibody detection was by anti-mouse-horseradish peroxidase secondary antibody (1:10,000; Amersham Biosciences, Piscataway, NJ) followed by enhanced chemiluminescence (ECL) (Pierce Chemical, Rockford, IL). Blotting for p150glued was with a mAb (Transduction Laboratories, San Jose, CA). Blot overlay assays were performed as described above, except the proteins eluted from the immunoprecipitation were separated by SDS-PAGE and transferred to polyvinylidene difluoride, and the transferred proteins were renatured on the blot by using serial guanidine dilutions. They were then blocked with BSA and probed with biotinylated EB1 (Pierce Chemical) (biotinylated according to the manufacturer's instructions), followed by detection using streptavidin-horseradish peroxidase and ECL detection. Quantitation of Coomassie-stained and ECL-detected bands was done using the quantitation feature of the public domain software NIH Image (available at http://rsb.info.nih.gov/nihimage/).

\section{Human EB1 Protein Expression, Purification, and Labeling}

Human EB1 (Berrueta et al., 1998) was cloned as a BamHI-HindIII fragment into the pet28A (Novagen, Madison, WI) to fuse six histidines to the $\mathrm{N}$ terminus of EB1, and 6His-human EB1 was expressed in BL21 PLysS cells (Novagen) and purified using nickelagarose beads (Sigma-Aldrich) according to the manufacturer's instructions. The protein was labeled with the succinimidyl ester of Alexa $^{488}$ (Molecular Probes, Eugene, OR), according to the manufacturer's instructions, eluted in PBS, and flash frozen in liquid nitrogen in $5-10-\mu l$ aliquots before use. Similar results for protein localization studies were obtained with Alexa ${ }^{488}$ and Alexa ${ }^{594}$ labeled EB1.

\section{Tubulin Purification and Labeling}

Tubulin was purified from calf brain by two cycles of polymerization-depolymerization and phosphocellulose chromatography as 
Table 1. Microtubule dynamics in Xenopus egg extracts with endogenous and additional EB1

\begin{tabular}{|c|c|c|c|c|c|}
\hline \multirow[b]{2}{*}{ Parameter } & \multicolumn{3}{|c|}{ CSF } & \multicolumn{2}{|c|}{ Interphase } \\
\hline & $1 \times \mathrm{EB} 1$ & $2.5 \times \mathrm{EB} 1$ & $8.5 \times \mathrm{EB} 1$ & $1 \times \mathrm{EB} 1$ & $2.5 \times \mathrm{EB} 1$ \\
\hline Polymerization rate & $10.0(10.7-13.3)$ & $8.7(9.0-10.4)$ & $10.4(10.3-11.5)$ & $6.4(6.7-8.9)$ & $14.1(13.8-14.3)$ \\
\hline Depolymerization rate & $9.1(9.2-11.2)$ & $5.9(4.3-8.1)$ & n.a. & $12.5(10.5-18.4)$ & $7.0(4.3-9.8)$ \\
\hline Catastrophe frequency & 0.37 & 0.05 & 0 & 0.12 & 0.05 \\
\hline Rescue frequency & 0.16 & 1.60 & 2.80 & 0.39 & 0.99 \\
\hline Time polymerizing, $\%$ & 51 & 90 & 96 & 52 & 78 \\
\hline Time depolymerizing, \% & 19 & 3 & 0 & 5 & 1 \\
\hline Time pausing, $\%$ & 30 & 6 & 3 & 43 & 21 \\
\hline
\end{tabular}

Measurements were made during CSF arrest and interphase arrest. $1 \times$ EB1 refers to extract without added EB1, 2.5 $\times$ EB1 refers to extract with additional $0.34 \mu \mathrm{M}$ EB1, and 8.5 $\times$ EB1 refers to extract with additional $1.72 \mu \mathrm{M}$ EB1. Rates of polymerization and depolymerization are in microns per minute (with 95\% confidence intervals). Frequencies of rescue and catastrophe are in events per minute. For CSF $1 \times, 76$ microtubules were analyzed for $133 \mathrm{~min}$; for CSF $2.5 \times, 131$ microtubules were analyzed for $397 \mathrm{~min}$; for CSF $8.5 \times, 114$ microtubules were analyzed for $341 \mathrm{~min}$, for interphase $1 \times, 28$ microtubules were analyzed for $101 \mathrm{~min}$; and for interphase $2.5 \times, 27$ microtubules were analyzed for $61 \mathrm{~min}$.

n.a., not applicable because no depolymerizations were observed.

described previously; aliquots were thawed and labeled with tetramethyl- or- X-rhodamine (Molecular Probes) according to Hyman et al. (1991).

\section{Imaging of Centrosomally Nucleated Microtubule Asters}

Centrosomes were purified from Chinese hamster ovary $(\mathrm{CHO})$ cells as described previously (Mitchison and Kirschner, 1986) and stored at $-80^{\circ} \mathrm{C}$. Microtubule asters were nucleated in EB1-depleted or mock-depleted crude extracts containing centrosomes and a final concentration of $\sim 5 \mu \mathrm{M}$ rhodamine-labeled bovine (calf) brain tubulin and incubated for $10-20 \mathrm{~min}$ at a room temperature of $\sim 23^{\circ} \mathrm{C}$. For add-back experiments, phosphate-buffered saline or EB1 (10 $\mathrm{ng} / \mu \mathrm{l}$ final concentration) was added to the reaction before room temperature incubation. Additions were $<10 \%$ of the reaction volume. Reactions were diluted into BRB80 (80 mM K-PIPES, $1 \mathrm{mM}$ $\mathrm{MgCl}_{2}, 1 \mathrm{mM}$ EGTA, pH 6.8) in 30\% glycerol and centrifuged at $6000 \times g$ for $10 \mathrm{~min}$ through a $5-\mathrm{ml}$ cushion of $40 \%$ glycerol in BRB80 onto poly-lysine-precoated coverslips (Desai et al., 1998). Images of fixed asters were acquired on an upright Nikon E-600 or E-800 microscope equipped with a cooled charge-coupled device camera (Princeton Instruments, Trenton, NJ) by using MetaMorph software (Universal Imaging, West Chester, PA). Aster radii were measured from digital images (radii were used instead of diameters because some asters were rendered asymmetrical by the pelleting and fixation procedures). In each case, three or four independent experiments gave similar results. For determining the ratio of Alexa ${ }^{488}$-EB1 on microtubule tips vs. walls, the ratio of the maximum comet fluorescence was compared with the mean wall fluorescence for each of 36 microtubules, and the mean $( \pm \mathrm{SD})$ was calculated from these ratios. EB1 fluorescence on microtubules nucleated in CSF vs. interphase extracts was compared under identical microscope and camera settings by using precise EB1 and tubulin concentrations, from the same aliquot in each case.

\section{Microtubule Dynamics Microscopy and Analysis}

Frozen extracts were thawed on ice and incubated with $\mathrm{CHO}$ centrosomes, tetramethyl-rhodamine-tubulin, and Alexa ${ }^{488}$-labeled 6 His-human EB1 protein in a final volume of $20 \mu \mathrm{l}$, with additives accounting for $<10 \%$ of the final reaction volume. Human EB1 was added to a final concentration of $0.34 \mu \mathrm{M}$ (total of Xenopus plus human EB1 2.5×) or $1.72 \mu \mathrm{M}$ (total of Xenopus plus human EB1
$8.5 \times$ ), as noted in Table 1. Coverslip squashes were made with 1.2 $\mu l$ of the reaction and imaged immediately at a room temperature of $23^{\circ} \mathrm{C}$. Time-lapse series were taken at $100 \times / 1.4$ numerical aperture magnification on an upright E800 microscope (Nikon, Tokyo, Japan) equipped with a CS10 spinning disk confocal head (PerkinElmer Life Sciences, Boston, MA) and acquired digitally by an Orca ER cooled charge-coupled device camera (Hamamatsu Photonics, Bridgewater, NJ) by using MetaMorph software.

Microtubule dynamics was measured in the time-lapse images by using the MetaMorph "track points" function, assigning the origin to the centrosome. On paused microtubules with extensions, the length was measured to the base of the extension. Coordinates were entered into an Excel (Microsoft, Redmond, WA) spreadsheet, and mean distance from the centrosome was calculated using $X^{2}+Y^{2}=$ $\mathrm{Z}^{2}$. Microtubule life-history tables and polymerization and depolymerization rates were plotted using CricketGraph (Computer Associates, Islandia, NY). Rates that failed to fulfill $\mathrm{R}^{2}>0.9$ were omitted from the analysis. Excel (Microsoft) was used for statistical analysis of the microtubule dynamics parameters.

\section{Fluorescence Recovery after Photobleaching of EB1}

Extracts containing centrosomes, rhodamine-tubulin, and Alexa ${ }^{488}$ labeled EB1 were prepared as described above. Photobleaching was performed using a $25-\mathrm{ms}$ exposure to a $100-\mathrm{mW}, 488-\mathrm{nm}$ beam from an Ar-ion laser (Spectra Physics, San Jose, CA) expanded and focused by a $60 \times / 1.4$ Plan Apo objective on the field diaphragm of an inverted TE300 microscope (Nikon). Epifluorescence images were acquired with an Orca ER camera (Hamamatsu Photonics) by using the MetaMorph software "stream acquisition" feature, which directly streams data to memory. For background subtraction, the brightness of a 5 by 5 pixel area was integrated for the entire time series, and three squares of the same size were subtracted from the bleached spot as well as a control, nonbleached spot on the same microtubule. To control for photobleaching from the mercury arc lamp during image acquisition, a reference integrated brightness value was obtained from three squares placed on fluorescent microtubules distant from the bleached spot (control nonbleached spot). The value of the bleached spot was divided by the value for the control nonbleached spot at each time point in the series, as was done in Maddox et al. (2000). The time dependence of the bleached signal was described by the function $F=\left(F_{R}-F_{b l c h}\right)\left(1-e^{-k t}\right)+$ $\mathrm{F}_{\mathrm{blch}}$, where $\mathrm{F}$ is the fluorescence signal corrected for background fluorescence, $F_{R}$ is the recovered fluorescence, $k$ is fluorescence 
recovery constant, and $\mathrm{F}_{\mathrm{blch}}$ is the fluorescence at the time of bleaching (Bulinski et al., 2001). The $t_{1 / 2}$ is calculated as $\ln (2) / \mathrm{k}$. The average percentage of recovery $\left(\mathrm{F}_{\mathrm{R}}-\mathrm{F}_{\mathrm{blch}}\right) /\left(\mathrm{F}_{\text {preblch }}-\mathrm{F}_{\mathrm{blch}}\right), 70 \pm$ $25 \%$, was similar for CSF and interphase extracts. The remaining $30 \%$ of fluorescence not recovered likely represents more stably bound or nonexchangeable EB1. The large degree of variability in fluorescence recovery after photobleaching (FRAP) half-lives in CSF and interphase extracts ( $\geq 50 \%$ in both cases) is consistent with values found by other investigators (Olmsted et al., 1989; Maddox et al., 2000; Bulinski et al., 2001).

\section{Correlation of Comet Length and Polymerization Rate: Regression Analysis}

Sections of time lapses during which rates were stable for several time points were chosen for a variety of microtubules on the same coverslip. For this set of microtubules, comet spatial distribution and temporal decay was determined by regression analysis by using Kaleidograph (Synergy Software, Reading, PA).

\section{Microtubule Copelleting and Pure Tubulin Polymerization Assays}

Binding of EB1 to in vitro-assembled microtubules was assayed in a copelleting assay. Briefly, $300 \mathrm{nM}$ EB1 was precleared by centrifugation and incubated with Taxol-stabilized microtubules (BristolMeyers-Squibb, New York, NY) in a titration from 0.05 to $10 \mu \mathrm{M}$ for $15 \mathrm{~min}$ at $37^{\circ} \mathrm{C}$. The reaction was pelleted at $60,000 \mathrm{rpm}$ for $30 \mathrm{~min}$ at $20^{\circ} \mathrm{C}$ in a TLA 100 rotor (Beckman Coulter, Fullerton, CA). Pelleted proteins were resuspended in SDS-PAGE sample buffer and analyzed by densitometry of a Coomassie-stained SDS-PAGE. The $K_{\mathrm{d}}$ was determined from the best fit line.

A visual assay was used to test whether EB1 could enhance the polymerization of microtubules in vitro. GMPCPP microtubule seeds (brightly labeled using twice-cycled tubulin in a ratio of 1:3 X-rhodamine-labeled tubulin to unlabeled tubulin, polymerized with $1 \mathrm{mM}$ GMPCPP (Hyman et al., 1992), pelleted, and resuspended in BRB80) were used to nucleate the polymerization of microtubule extensions (dimly labeled using twice-cycled tubulin in a ratio of 1:22 X-rhodamine-labeled tubulin to unlabeled tubulin) at a tubulin concentration of $20 \mu \mathrm{M}$ in BRB80 with $1 \mathrm{mM}$ GTP, $1 \mathrm{mM}$ dithiothreitol, $100 \mathrm{mM} \mathrm{NaCl}$, and $100 \mathrm{ng} / \mu \mathrm{l}$ EB1 protein or control buffer. At 2, 4, and $6 \mathrm{~min}$, microtubules were fixed with $0.2 \%$ gluteraldehyde in BRB $80 / 64 \%$ glycerol, imaged immediately, and the lengths of the dim extensions were measured using MetaMorph software. Mean lengths were similar in the presence and absence of EB1 $(n=65-272$ microtubules for each condition).

\section{RESULTS}

\section{Immunodepletion of EB1 from Xenopus Egg Extracts}

Before manipulating the levels of EB1 in Xenopus egg extracts, we determined the endogenous level of EB1 in the extracts in comparison with known amounts of bacterially expressed EB1. The amount of EB1 in both CSF and interphase extracts was $\sim 8 \mathrm{ng} / \mu \mathrm{l}(\sim 0.27 \mu \mathrm{M})$, almost 100 -fold less than the amount of endogenous tubulin (18 $\mu \mathrm{M}$; Parsons and Salmon, 1997) (Figure 1a; our unpublished data). Using a $\mathrm{mAb}$ generated against human EB1, we were able to immunodeplete $>95 \%$ of detectable EB1 from the extracts (Figure 1b; our unpublished data). Depletion of EB1 from both CSF and interphase extracts also removed several EB1interacting proteins from the extracts (Figure 1c). These were identified by LC/MS as components of cytoplasmic dynein and the dynactin activator complex. The interaction between EB1 and dynein/dynactin seemed to be mediated by the p150glued component of dynactin, because EB1 bound directly to p150glued in a blot overlay assay (Figure 1d). Although substantial amounts of dynein/dynactin bound EB1, at least $50 \%$ of p150glued remained in the extract, as determined by Western blotting (our unpublished data). By Western blotting, we did not detect APC in the EB1 immunoprecipitates (our unpublished data).

\section{EB1 Is a Major Regulator of Microtubule Dynamics}

To test the role of EB1 in microtubule dynamics, we first assayed microtubule length at fixed time points in Xenopus egg extracts, by using $\mathrm{CHO}$ centrosomes to nucleate microtubules and rhodamine-labeled tubulin to visualize asters. As shown in Figure 1, e and f, asters in EB1-immunodepleted CSF extracts were barely visible due to their small diameter, whereas mock-depleted extracts were competent to nucleate large asters. The mean aster radius was $2.7 \pm 1.1$ $\mu \mathrm{m}$ in EB1-depleted extracts $(\mathrm{n}=83)$, compared with $14.2 \pm$ $5.3 \mu \mathrm{m}$ in mock-depleted extracts $(\mathrm{n}=113)$, a 5.3 -fold difference (3-5-fold greater aster radii in EB1-depleted vs. mock-depleted extracts was confirmed in three additional experiments). In contrast, depletion of EB1 from interphase extracts did not reduce microtubule length (Figure 1, g and h). In interphase extracts, mean aster radius was $7.9 \mu \mathrm{m}$ in both EB1 $(\mathrm{n}=20 ; \mathrm{SD}=2.0)$ and mock-depleted extracts $(\mathrm{n}=$ 28 ; $\mathrm{SD}=2.1)$ (similar mean aster radii were confirmed in EB1-depleted vs. mock-depleted extracts in three additional experiments). Thus, EB1 immunodepletion substantially reduced microtubule length in CSF but not interphase extracts.

To test whether the effect we saw on microtubule length was due to depletion of EB1 rather than partial depletion of dynein/dynactin or another protein, we added back human EB1, expressed and purified from Escherichia coli, to near endogenous levels $(0.23 \mu \mathrm{M})$. Human EB1 was used because we were unable to successfully clone Xenopus EB1 for these studies. As shown in Figure 1, i-1, the microtubule length reduction caused by EB1 depletion was fully complemented by the readdition of EB1 to the extracts. Readdition of EB1 to the EB1-depleted extracts increased aster radius 4.9-fold, returning them to the size typical of asters in mock-depleted extracts. Doubling the amount of EB1, by adding EB1 to mock-depleted extracts, resulted in further lengthening of microtubules, increasing aster radius 2.3-fold ( $\mathrm{n}=104$ measurements in two independent experiments). The results described here were also true for the Alexa ${ }^{488}$-labeled human EB1 used for subsequent fluorescence microscopy studies (our unpublished data). These assays indicate that EB1 was the critical component for microtubule stability removed by the immunoprecipitation.

\section{EB1 Is an Antipause, Anticatastrophe Factor}

Because the fixed time-point assays showed a dramatic effect of EB1 on microtubule length, we proceeded to analyze life histories of individual microtubules to determine which parameters of dynamic instability were modified by EB1. Microtubules in EB1-depleted extracts were too short to measure reliably, so for CSF extracts, we compared endogenous levels of EB1 with two concentrations of added EB1. On the basis of our stoichiometry analysis, these corresponded to $1 \times$ (endogenous EB1 only), $2.5 \times$ (endogenous plus $0.34 \mu \mathrm{M}$ EB1), and $8.5 \times$ (endogenous plus $1.7 \mu \mathrm{M}$ EB1). We found a 

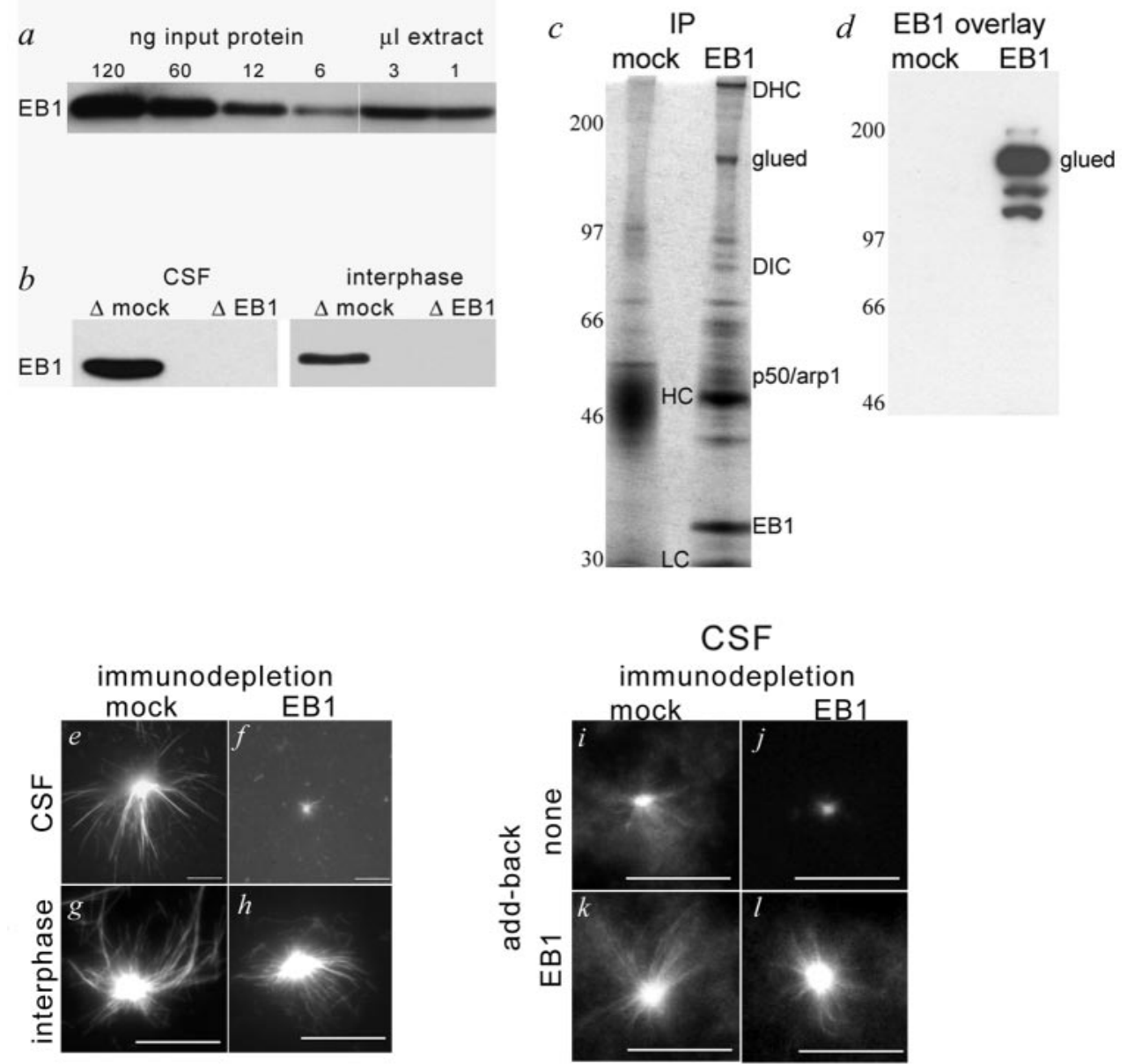

Figure 1. EB1 stabilizes microtubules in Xenopus egg extracts. (a) Determination of EB1 concentration in extracts. Anti-EB1 Western blot shows a titration of known input amount of bacterially expressed EB1 vs. a known volume of extract. We estimated the concentration of EB1 in extracts to be $\sim 8 \mathrm{ng} / \mu \mathrm{l}$. (b) EB1 can be immunodepleted from extracts. AntiEB1 Western blot of CSF and interphase extracts mock immunodepleted using mouse IgG or immunodepleted of EB1 show $>95 \%$ removal of EB1 protein. (c) Coomassie-stained gel of proteins eluted from the EB1 immunoprecipitation. Identities of the eluted proteins were determined by LC/ MS of bands cut from the gel and were independently established by Western blotting. Dynein heavy chain (DHC), p150glued (glued), dynein IC (DIC), p50dynamitin (p50), arp1/centractin (arp1), and EB1 were present in the EB1 but not the mock immunoprecipitation. The immunoprecipitating antibody heavy chains (HC) and light chains (LC) are indicated. (d) EB1 and mock immunoprecipitations probed with biotinylated EB1 show direct binding of EB1 to the p150glued dynactin component. The band marked "glued" was identified as biotinylated EB1 bound to 150glued because it exactly comigrated with a p150glued band from the EB1 immunoprecipitation, run on a different portion of the same gel and directly stained with Coomassie as in c. The lower intensity bands near the p150glued band have not been characterized. $(\mathrm{e}-\mathrm{h})$ Immunodepletion of EB1 results in shorter microtubules in CSF but not interphase, extracts. Representative centrosome-nucleated asters were visualized by rhodamine-tubulin fluorescence in mock-immunodepleted and EB1-immunodepleted crude extracts (see MATERIALS AND METHODS). (e and f) CSF extracts. (g and h) Interphase extracts. (e and g) Mock immunodepletion. (f and h) EB1 depletion. The CSF and interphase experiments are separate experiments and are not directly comparable. See text for quantitation. (i and 1) Readdition of EB1 to depleted CSF extracts reveals the importance of EB1 for microtubule stability. Examples of centrosome-nucleated asters visualized by rhodamine-tubulin fluorescence are shown; separate experiments show add-back of EB1 to mock depleted and EB1-depleted extracts. These experiments are separate from the experiments in e and f and are not directly comparable because different extract preparations varied slightly in their abililty to form asters. (i) Mock depletion, no EB1 add-back. (j) EB1 depletion, no EB1 add-back. (k) Mock depletion, EB1 add-back. (l) EB1 depletion, EB1 add-back. See text for quantitation. Bars, $10 \mu \mathrm{m}$.

dose-response effect of EB1 on parameters of microtubule dynamic instability (Table 1). These included a decrease in the catastrophe frequency (from $0.4 / \mathrm{min}$ for endogenous to $0.1 / \mathrm{min}$ for $2.5 \times$ and $0 / \mathrm{min}$ for $8.5 \times)$, and an increase in the rescue frequency (from $0.2 / \mathrm{min}$ to $1.6 / \mathrm{min}$ and $2.8 / \mathrm{min}$ ). The polymerization rate was essentially unaffected, whereas the depolymerization rate was slowed from 9.1 to $5.9 \mu \mathrm{m} /$ min. Of note, we did find significant variation in polymerization rates among microtubules within the same extract preparation, and for a single microtubule during its lifetime. Pause time (lack of statistically significant polymerization or depolymerization $>3-4$ data points) was also decreased in the presence of excess EB1 (from 30 to 6 to 3\%). This number may underestimate the pause frequency, because micropauses of $5 \mathrm{~s}$ or less would not be counted in this analysis.
This combination of changes led to an increase in the time spent polymerizing (from 51 to 91 to $96 \%$ ) and a reduction in the time spent depolymerizing (from 19 to 3 to $0 \%$ ). The 5 -s recording interval we used may have made our measurements insensitive either to numerous microexcursions or brief pauses, but the dose-response relationship we observed suggests that we were recording enough of each type of dynamic instability state to measure a true biological effect. The net result of EB1 addition was to decrease catastrophe frequency and pause time, to increase rescue frequency and polymerization time, and to slow depolymerization rate, effects that together stabilized microtubules.

For interphase extracts, similar effects of EB1 addition were observed. Addition of the highest EB1 concentration 
$a$ interphase
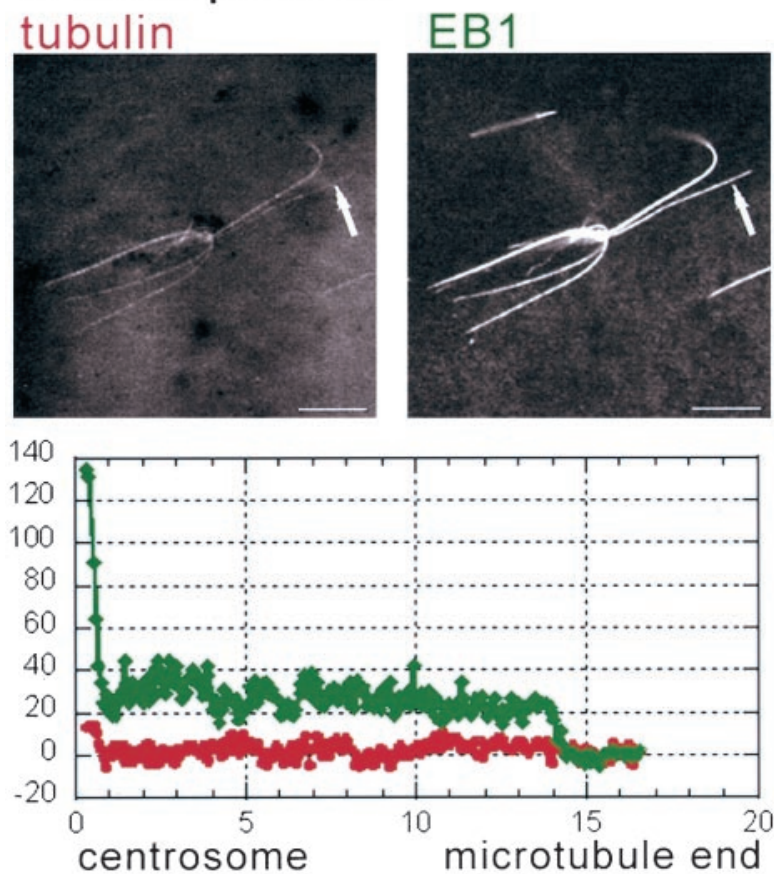

\section{$b$ CSF}
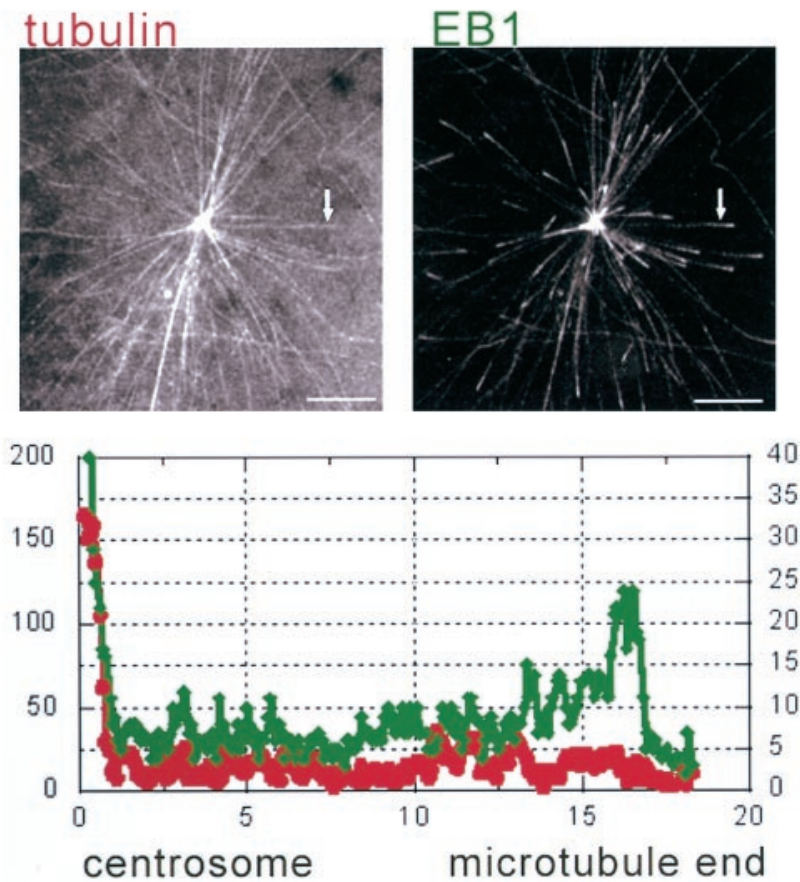

$c$
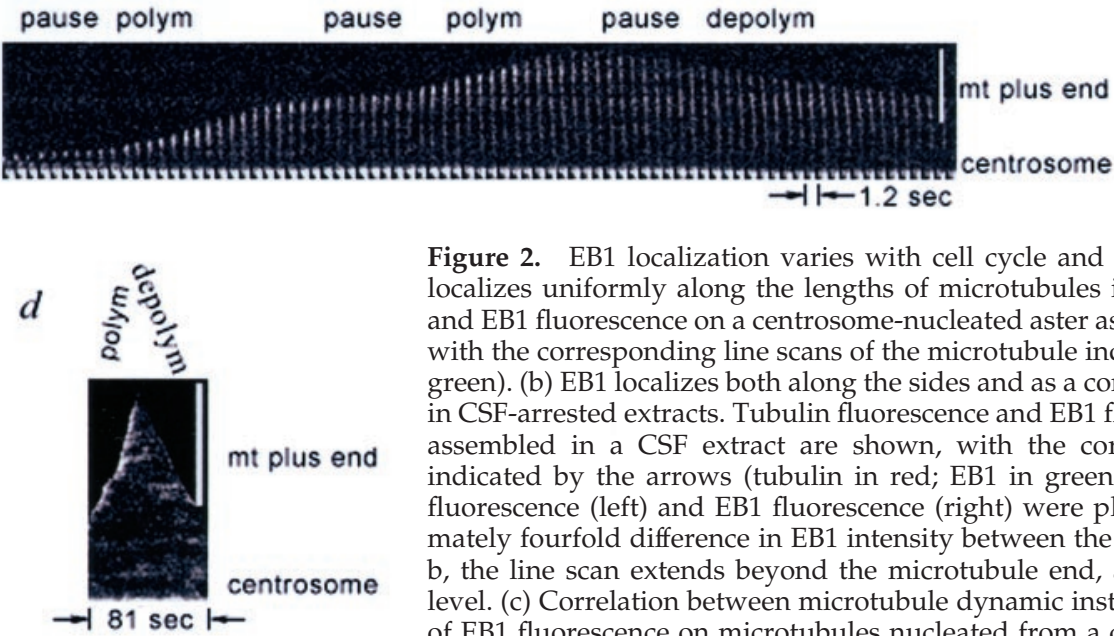

Figure 2. EB1 localization varies with cell cycle and microtubule polymerization status. (a) EB1 localizes uniformly along the lengths of microtubules in interphase extracts. Tubulin fluorescence and EB1 fluorescence on a centrosome-nucleated aster assembled in an interphase extract are shown, with the corresponding line scans of the microtubule indicated by the arrows (tubulin in red; EB1 in green). (b) EB1 localizes both along the sides and as a comet at the tips of polymerizing microtubules in CSF-arrested extracts. Tubulin fluorescence and EB1 fluorescence on a centrosome-nucleated aster assembled in a CSF extract are shown, with the corresponding line scans of the microtubule indicated by the arrows (tubulin in red; EB1 in green). For this line scan, the y-axes for tubulin fluorescence (left) and EB1 fluorescence (right) were plotted separately to emphasize the approximately fourfold difference in EB1 intensity between the microtubule tip and the wall. In both a and $\mathrm{b}$, the line scan extends beyond the microtubule end, and EB1 fluorescence drops to background level. (c) Correlation between microtubule dynamic instability and comet appearance. One example of EB1 fluorescence on microtubules nucleated from a centrosome in CSF extracts is shown from a time lapse taken at 1.5-s intervals. Microtubule polymerization, pausing, and depolymerization are indicated. A different microtubule undergoing polymerization and depolymerization is illustrated in a kymograph. The position of the centrosome and microtubule end is indicated. Note the appearance of the EB1 comet during polymerization and its disappearance during pausing and depolymerization. The images are scaled and contrast enhanced to show the low level EB1 fluorescence along the microtubule length. Bars, $10 \mu \mathrm{m}$.

$(8.5 \times)$ produced severe microtubule bundling that prohibited reliable tracking of individual microtubules. Similar bundling has been observed using overexpressed protein in interphase tissue culture cells (Bu and $\mathrm{Su}, 2001)$. We were, however, able to measure microtubule dynamics in unmodified extracts vs. extracts with addition of EB1-2.5 $\times$, although we did so for fewer microtubules than for the CSF arrest. Essentially, the effects of EB1 addition on catastrophe and rescue frequencies, depolymerization rate, and time distribution were similar to those seen in CSF extracts. The one significant difference was that in interphase extracts, EB1 increased the polymerization rate from 6.4 to $14 \mu \mathrm{m} / \mathrm{min}$. Thus, although immunodepletion of EB1 from interphase extracts did not dramatically reduce microtubule length, EB1 addition decreased catastrophes and pauses, increased rescues and polymerization, and increased polymerization rate. 


\section{EB1 Localization on Microtubules Is Cell Cycle Regulated in Xenopus Egg Extracts}

To determine its localization, we added EB1 that was directly labeled with the Alexa ${ }^{488}$ fluorophor to Xenopus egg extracts, during interphase and CSF arrest (Figure 2, a and b). We saw a dramatically different pattern of localization under these two conditions. In CSF extracts, EB1 localized in a bright comet-shaped streak at the plus ends of polymerizing microtubules, and in a fainter signal along the walls ${ }^{1}$ of all microtubules (Figure 2b). Of note, the level of EB1 we added was low enough that the fluorescence on the microtubule was somewhat speckled rather than completely uniform. The peak intensity of the comet was $4 \pm 2$-fold brighter than the microtubule wall $(n=36)$. When a microtubule plus end underwent a catastrophe, the EB1 comet was lost, whereas the faint uniform signal on the wall persisted unchanged in intensity. During microtubule pauses, loss of the EB1 signal occurred coincident with the pause, but occasionally preceded or followed it (an example of the former is seen in Figure 2c). These localization characteristics are illustrated in Figure 2, c and d. In the CSF extract system, free microtubules were released from centrosomes and nucleated spontaneously, as visualized by EB1 fluorescence. In cases where both ends of a free microtubule were visible, we did not see EB1 comets at the minus end (polarity was inferred from the presence of dynamic instability, absent from minus ends in Xenopus egg extracts; Gard and Kirschner, 1987; Parsons and Salmon, 1997). The bright EB1 fluorescence at the centrosome is therefore likely to be due to labeling of the plus ends of very short microtubules or of the centrosome itself, rather than microtubule minus ends.

In extracts made from interphase-arrested eggs, we saw a dramatically different pattern of EB1 localization. EB1 was distributed uniformly along the entire length of the microtubule, with occasional speckles visible (Figure 2a). This pattern persisted even when we lowered the concentration of added EB1 to 25\% of that used in CSF extracts (our unpublished data). In addition to differing from the localization seen in CSF extracts, this localization differs from the comet-like pattern that others and we have seen in interphase tissue culture cells. Moreover, the difference was more dramatic in arrested extracts than in extracts traversing the cell cycle after addition of calcium (Tirnauer and Grego, unpublished data). Compared with the intensity of EB1 on microtubule walls in CSF extracts, the intensity of EB1 fluorescence on interphase microtubules was $3 \pm 2$-fold brighter $(n=32)$. Consistent with greater EB1 binding, the EB1 speckles were less pronounced on microtubules in interphase extracts than on microtubules in CSF extracts, for the same concentration of EB1 added and the same exposure time (the contrast of fluorescent speckles decreases with higher concentrations of bound fluorescent protein; Waterman-Storer and Salmon, 1999). Comparison of the CSF and

\footnotetext{
1 "Older microtubule lattice" and "newer lattice" are technically more accurate. We do not mean to imply that EB1 interacts exclusively with the ultimate sheet of protofilaments on the microtubule plus end, but rather the region near the end composed of newly polymerized subunits. We use "microtubule plus end or tip" and "microtubule wall" herein for convenience.
}

interphase image intensities shows that the brightest signal on the CSF comets was similar to the uniform wall signal on interphase microtubules. Thus, the ratio of EB1 tip to wall binding in CSF extracts was due to a reduction in microtubule wall binding rather than to an increase in microtubule tip binding.

\section{EB1 Binds to Microtubule Plus Ends and Walls by Separate Mechanisms}

The differences in the spatial distribution of EB1 along the lengths of microtubules in CSF extracts and in the pattern of EB1 distribution between CSF and interphase arrest suggested different mechanisms of EB1 binding. We addressed these microtubule wall vs. tip-binding mechanisms separately. First, to probe the dynamics of EB1 binding to the walls of intact microtubules, we performed FRAP experiments on Alexa ${ }^{488}$-EB1 protein bound to microtubule walls. We added equal amounts of labeled EB1 to microtubule asters in CSF- and interphase-arrested extracts and used a focused laser to bleach a spot of EB1 fluorescence on a microtubule wall. We recorded recovery of fluorescence, as EB1 with bleached fluorophors dissociated and EB1 with unbleached fluorophors associated with sites on the microtubule wall. In both cell cycle arrest states, we found rapid recovery of the majority of EB1 fluorescence (to $70 \%$; see MATERIALS AND METHODS). However, as shown in Figure 3, there was a significant difference between the two cell cycle states, with a recovery half-life of $3.6 \pm 2.4 \mathrm{~s}$ in CSF extracts $(n=21)$ and $12.0 \pm 6.5 \mathrm{~s}$ in interphase extracts $(\mathrm{n}=$ 21). EB1 thus bound to the walls of intact microtubules by a microtubule polymerization-independent mechanism, with exchange occurring more rapidly at binding sites on the microtubule during CSF arrest. Because the change in steady-state binding between CSF and interphase (threefold greater in interphase) was similar to the change in dissociation rate inferred from the FRAP experiments (threefold slower dissociation in interphase), we conclude that EB1 most likely has the same effective concentration and association rate in CSF arrest and interphase and that the different targeting is regulated only by the dissociation rate.

To test whether EB1 dissociation from the polymerizing microtubule end was similar to dissociation from the wall, we measured the decay of EB1 fluorescence over time. Fluorescence intensity at a single point on the microtubule wall was measured in sequential frames of a time-lapse series (Figure 4). When we analyzed fluorescence intensity vs. time, we found a close fit to an exponential decay, with a half-life of $2.6 \pm 1.5 \mathrm{~s}(\mathrm{n}=34)$, close to the value of $3.6 \mathrm{~s}$ measured by FRAP for EB1 dissociation half-life from the microtubule wall (Figure 4). This time-based analysis indicated that once EB1 bound the microtubule tip, it dissociated with first order rate kinetics. If polymerization rate were constant, a line scan along the comet, representing the distribution of EB1 in space, would also be expected to fit an exponential. When we plotted the intensity of EB1 fluorescence along the length of the comet, we found a less robust decay curve, and we measured a half-length of the comet of $0.7 \pm 0.3 \mu \mathrm{m}(\mathrm{n}=32)$ (Figure 4$)$. Of the two values, the temporal decay fits better with the mean polymerization rate of $10 \mu \mathrm{m} / \mathrm{min}$. The spatial decay curve may be affected by variable polymerization rates of the microtubule and non- 
Figure 3. EB1 fluorescence recovery after FRAP reveals rapid turnover of EB1 on microtubule sides. Alexa ${ }^{488}$-EB1 was added to the extract and laser photobleaching of the microtubule (bleach site marked by an arrowhead) was done during a digital stream acquisition of 1.2 $\mathrm{s} /$ frame. (a) Fluorescence recovery on a microtubule in CSF extract. The bleach mark is visible in the third frame. (b) Fluorescence recovery on a microtubule in an interphase extract. The bleach mark is visible in the second frame. In both montages, the 50 frames shown represent $60 \mathrm{~s}$. (c) Graphic depiction of the recovery half-lives for microtubules in CSF (green bars, $\mathrm{n}=21$ ) and interphase (blue bars, $\mathrm{n}=21$ ) extracts, with percentage of microtubules per recovery time shown for both conditions. The mean recovery half-life was $3.6 \pm 2.4 \mathrm{~s}$ for CSF extracts and $12.0 \pm 6.5 \mathrm{~s}$ for interphase extracts.
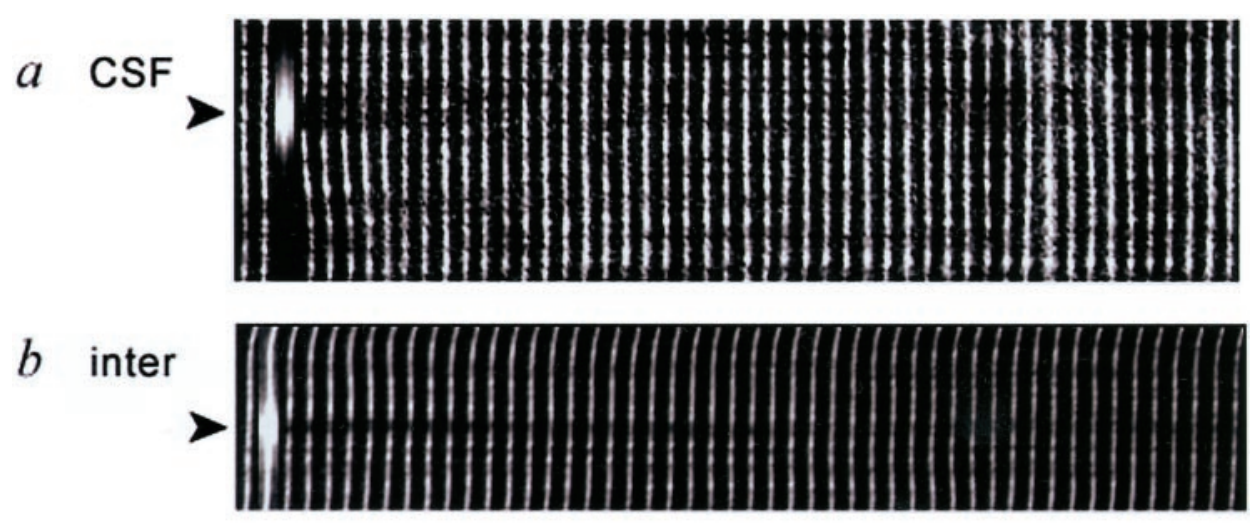

$c$

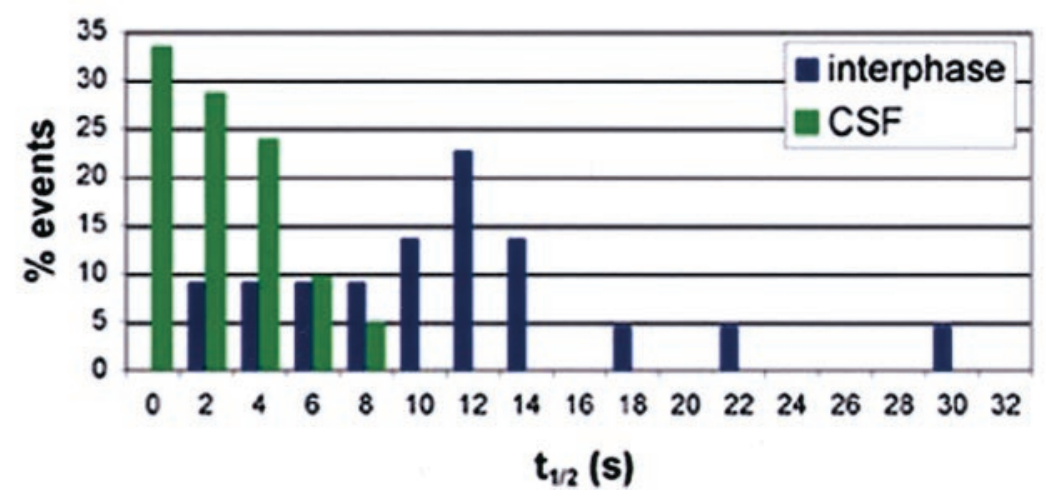

seconds, without a transition to depolymerization. Such pauses have been observed by others in vivo and in vitro (Walker et al., 1988; Tirnauer et al., 1999; Davis et al., 1993; Grego et al., 2001; Rusan et al., 2001), but their origins are obscure. On pausing microtubule ends visualized by EB1 fluorescence, the high signal-to-background ratio of EB1 binding allowed visualization of a novel structure not previously reported: curved filamentous extensions with the intensity expected for EB1 bound to a few tubulin protofilaments (Figure 6). The extensions appear to contain tubulin, although our images do not allow us to conclude this unequivocally (Figure $6 \mathrm{~b}$ ). The lengths of the filaments were variable, extending up to several microns in length (Figure 6c). They consistently curved outwards from the microtubule tip, with a curvature radius on the order of $1 \mu \mathrm{m}$ (Figure 6a). They occasionally polymerized, depolymerized, and, most strikingly, they were often observed to break off the microtubule, at which point the microtubule immediately commenced polymerization at near constant velocity. Thus, loss of these extensions correlated with bursts of polymerization. These observations suggest that formation of curved filaments at the microtubule tip contributed to nonproductive polymerization, perhaps by stabilizing a small group of protofilaments unable to undergo tubule closure, leading to microtubule pauses.

\section{In Vitro, EB1 Binds Weakly to Purified Microtubules}

We wished to determine whether the effect of EB1 on microtubules was direct or mediated by another factor. To 


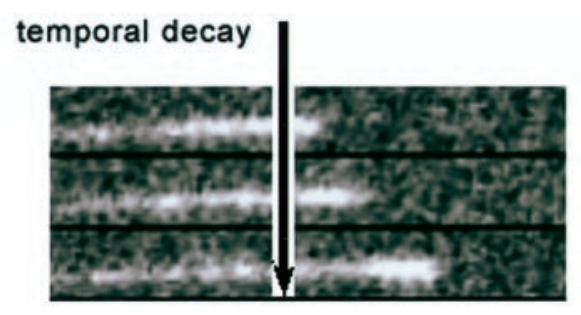

spatial distribution
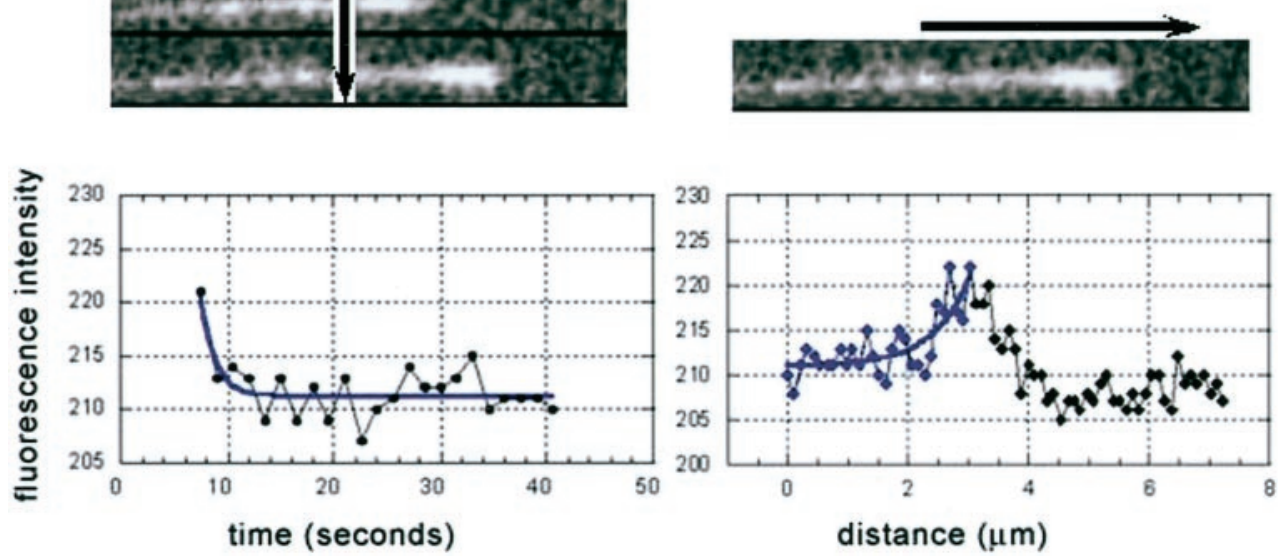

Figure 4. EB1 fluorescence intensity on polymerizing microtubule ends decays exponentially with time, consistent with a first order dissociation reaction. (a) Example of the fluorescence decay of Alexa ${ }^{488}$-EB1 at a single point on the microtubule over time. The image above the example shows the measurement axis (arrow indicates direction of measurement). The mean calculated decay half-time was $2.6 \pm 1.5 \mathrm{~s}(\mathrm{n}=34)$. (b) Example of the fluorescence distribution of $\mathrm{Al}-$ exa ${ }^{488}$-EB1 along the length of the microtubule at a single point in time. The microtubule is oriented with the distal ends facing toward the right (image above the example shows measurement axis, with arrow in direction of measurement). The mean calculated half-length of the comet was $0.7 \pm 0.3 \mu \mathrm{m}(\mathrm{n}=32)$. investigate this question, we tested whether EB1 could bind pure tubulin microtubules in a copelleting assay. As shown in Figure 7, bacterially expressed and purified EB1 bound to Taxol-stabilized microtubules assembled from bovine brain tubulin with a $K_{D}$ of $\sim 0.5 \mu \mathrm{M}$. This weak binding value is within the range previously reported (Berrueta et al., 1998) and consistent with our observation by FRAP that EB1 bound to the wall lattice of polymerized microtubules. To directly visualize the binding of EB1 to microtubules, we incubated Alexa ${ }^{488}$-labeled EB1 protein with X-rhodaminelabeled microtubules and imaged them by spinning disk confocal microscopy. Using this method, we saw faint binding along the lengths of microtubules but no specific binding to either end of the microtubule (our unpublished data). As has been described previously (Nakamura et al., 2001), we did not find gross effects of EB1 on microtubule polymerization by using pure EB1 and tubulin in vitro.

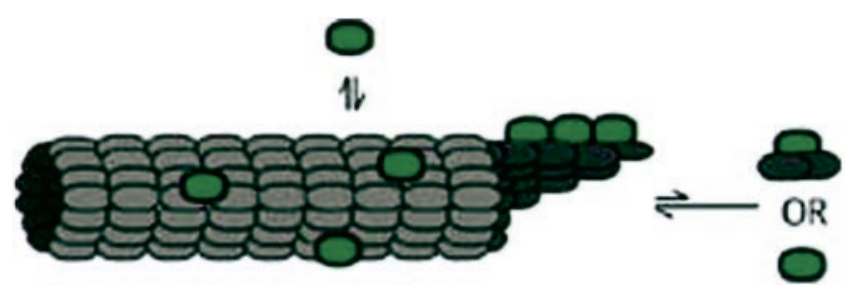

Figure 5. EB1 binding to microtubule walls and plus ends by separate mechanisms, with constant dissociation, would produce the observed localization pattern. Microtubule wall binding (vertical arrows) is in a steady state with dissociation, producing a faint uniform localization pattern. Near the polymerizing plus end, an additional targeting mechanism occurs (horizontal arrows), either by EB1 copolymerization with tubulin or by its recognition of a structural or chemical property of the end, which allows accumulation of EB1 in excess. Dissociation shapes the tail of the comet. The excess accumulation of EB1 as a comet shape predominates during CSF arrest.

\section{DISCUSSION}

\section{EB1 Is a Major Microtubule Stabilizer in Xenopus Egg Extracts}

Using a combination of immunodepletion and protein addition, we observed a major role for EB1 in regulating microtubule dynamic instability in Xenopus egg extracts. The reduction of microtubule length in CSF extracts depleted of EB1, compared with interphase, suggested EB1 may be more critical for microtubule stability during mitotic states than in interphase states. EB1 may be rate limiting for microtubule stabilization in CSF but not interphase, or, although unlikely, human EB1 (added back) may be more active than Xenopus EB1 (depleted) in the aster formation assay. Readdition of EB1 protein restored microtubule length in these depleted CSF extracts, implicating EB1, rather than the codepleted dynein/dynactin components, in stabilizing microtubules. The small size of asters upon EB1 depletion precluded us from asking whether EB1 protein depletion and EB1 protein addition produced exactly opposite effects. Addition of EB1 to undepleted extracts revealed a dose-dependent ability of EB1 to reduce pauses and catastrophes and to increase rescues, effects remarkably similar to those found in budding yeast (Tirnauer et al., 1999), except that the effects in yeast predominated during G1.

The highly conserved effects of EB1 on microtubule stability from yeast to mammals suggests a central role for EB1 in regulating microtubules (Tirnauer et al., 1999). We suggest that EB1, in addition to XMAP-215 and XKCM1, makes a major contribution to microtubule dynamics in Xenopus egg extracts, with the effect most pronounced during mitosis. It will be interesting to see how these regulators interact with each other. Like XMAP-215, EB1 may counteract the catastrophe-promoting activity of XKCM1. But EB1 lacks the in vitro activity seen for XMAP-215, so its effect on microtubule dynamics is likely to be indirect. Perhaps XMAP-215 in extracts is modified by EB1, although we did not find a physical interaction between EB1 and XMAP-215 in our 

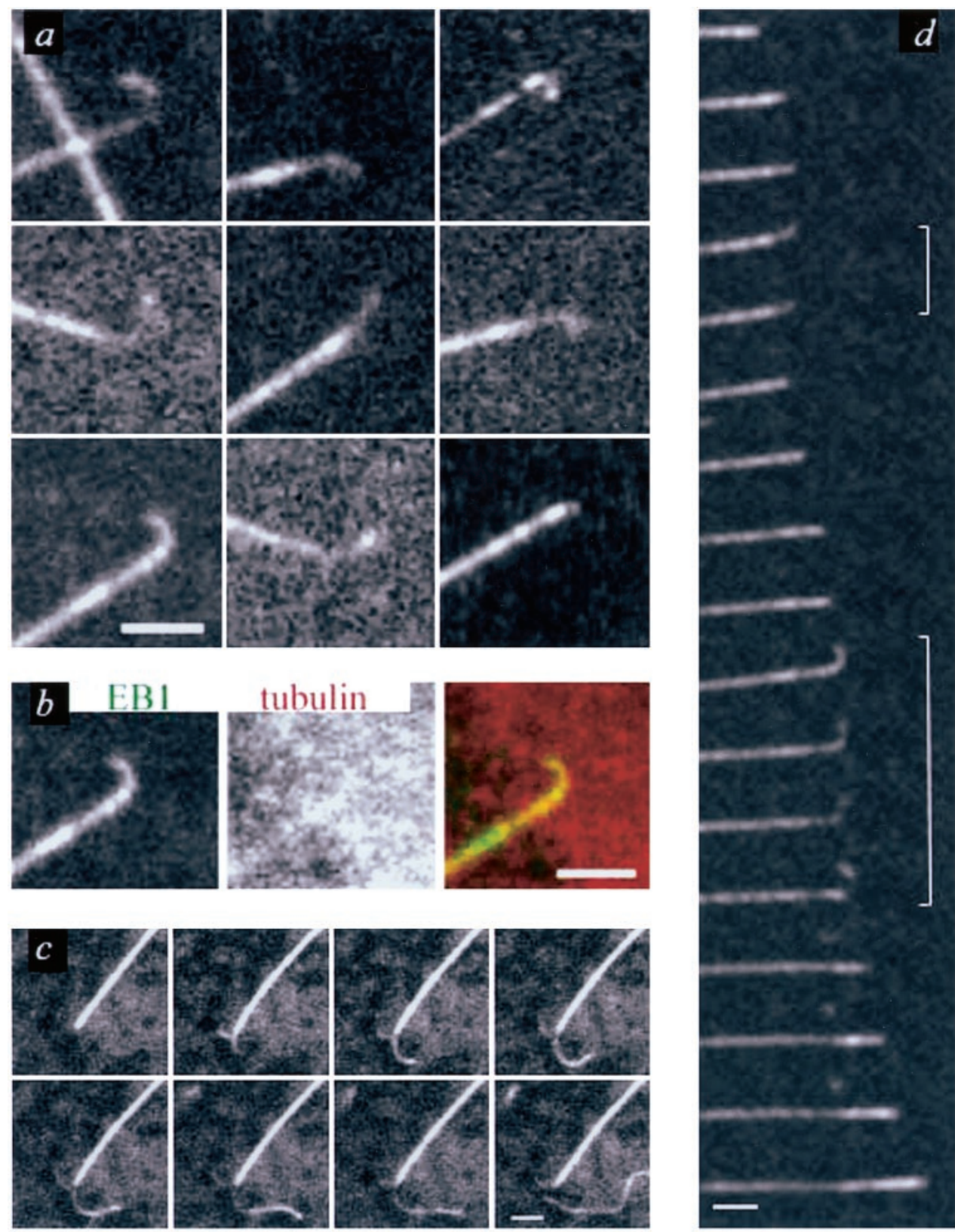

Figure 6. EB1 fluorescence reveals filamentous extensions on pausing microtubules. (a) Montage of filamentous extensions at the ends of microtubules in Xenopus egg extracts, visualized by Alexa ${ }^{488}$-EB1 fluorescence. The typical radius of curvature is $\sim 1 \mu \mathrm{m}$. (b) The extensions probably contain tubulin. For the same microtubule imaged by Alexa ${ }^{488}$-EB1 and rhodamine-tubulin fluorescence, the filamentous extension is barely visible by tubulin fluorescence. (c) Rarely, extensions bifurcated and/or elongated for several microns. Frames from a time lapse of EB1 fluorescence (interval $5 \mathrm{~s}$ ) reveal a bifurcating extension that grew several microns. (d) Typically, presence of the extensions correlated with microtubule pausing, and loss of the extension by breakage was followed by the abrupt onset of microtubule polymerization. Frames from a time lapse of EB1 fluorescence (interval $5 \mathrm{~s}$ ) show a microtubule that on two separate occasions paused with a protofilament extension (brackets). Loss of the protofilament extension was associated with polymerization, and during the second polymerization burst a piece of the detached extension can be seen beside the polymerizing microtubule. Bars, $2 \mu \mathrm{m}$.

immunoprecipitations. Alternatively, the in vitro reconstitution system may have been more similar to an interphase than a mitotic-like state.

In the Xenopus egg extract system, the highest stoichiometry binding partners for EB1 during $M$ phase and inter- phase were members of the dynein and dynactin complexes, mediated by direct binding between EB1 and the p150glued component of dynactin. In contrast, a functional rather than a physical interaction between the homologues of EB1 and dynein/dynactin has been shown in budding yeast. Yeast 


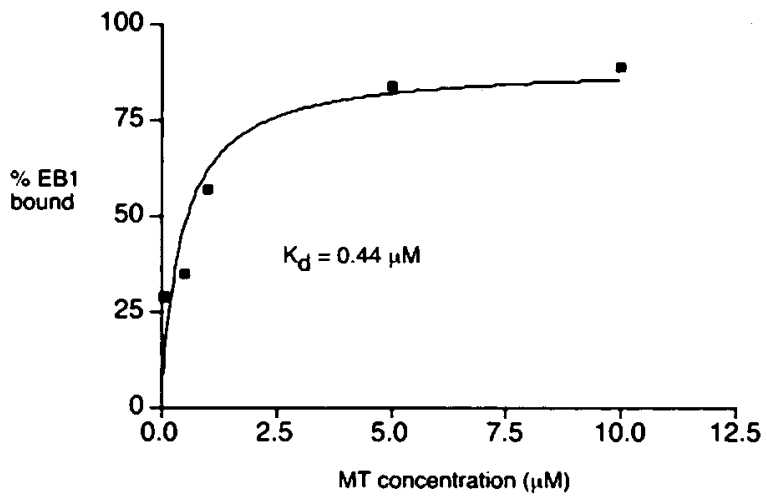

Figure 7. EB1 interacts with pure microtubules. Dissociation constant for EB1 binding to taxol-stabilized microtubules was calculated by best fit line to the data from an in vitro EB1-microtubulecopelleting experiment (see text for details).

EB1 and dynein/dynactin homologues both play roles in positioning the mitotic spindle, but they act in separate genetic pathways that seem to confer end-on microtubule attachment and sideways microtubule sliding along the bud cortex, respectively (Adames and Cooper, 2000; Schuyler and Pellman, 2001b). The discrepancy between the physical interaction in higher eukaryotic cells compared with the genetic interaction in budding yeast remains to be resolved.

\section{Does EB1 Stabilize Protofilament Extensions?}

In addition to demonstrating the effects of EB1 on microtubule dynamics in higher eukaryotes, our assays begin to suggest how these effects might be achieved. EB1 fluorescence revealed the appearance of loosely curved filamentous extensions on pausing microtubules. Loss of the extensions, often by breakage, correlated with the abrupt onset of polymerization, suggesting that the extensions were nonproductive for polymerization, yet nonpermissive for depolymerization. These extensions differed in radius of curvature by $\sim 2$ orders of magnitude from the 25-nm-diameter curls of individual GDP tubulin protofilaments induced during the depolymerization phase of microtubule dynamic instability (Mandelkow et al., 1991; Desai et al., 1999b; Tran et al., 1997; Arnal et al., 2000), and although we occasionally saw forked extensions, there was usually one, and never more than two per microtubule end. Colocalization with tubulin fluorescence suggested that these extensions contained tubulin protofilaments (the fluorescence was too weak to conclude unequivocally that these structures were present in the absence of EB1). These characteristics are consistent with small groups or pairs of laterally connected protofilaments extending from the microtubule plus end. Based on models correlating microtubule tip width with longitudinal curvature (Janosi et al., 1998), the extensions may be composed of many protofilaments. Their outward curl would make tubule closure nearly impossible, but their breakage might result in a shorter, unrolled sheet of protofilaments able to undergo tubule closure and polymerization. We do not know how these extensions are affected by EB1, but our observations that EB1 reduces microtubule pausing and increases rescues implies that EB1 might bias an equilibrium away from a pause configuration toward polymerization. This might be achieved by reducing the length or outward curl of these structures. Such effects could be achieved by enhancing lateral interactions among protofilaments, among other possibilities.

\section{EB1 Localizes Differently during CSF and Interphase Arrest}

In CSF Xenopus egg extracts, EB1 localized dimly along all microtubule walls, with an intense comet only on polymerizing microtubule ends. In contrast, in interphase extracts, EB1 fluorescence was intense along the entire microtubule. This cell cycle difference in EB1 localization deviates from what was seen by ourselves and others in tissue culture cells-comets on all polymerizing microtubule plus ends throughout the cell cycle. However, we did find comets to be shorter in mitotic tissue culture cells than in interphase cells (Tirnauer and Mitchison, unpublished data). Additionally, the cell cycle difference in Xenopus egg extracts was much more prominent during complete cell cycle arrest than when EB1 was imaged over the course of an activated extract cycle. Thus, we favor the interpretation that the inhibition of cyclin B synthesis during the prolonged interphase arrest altered the tubulin-microtubule equilibrium or the kinasephosphatase balance more severely than occurs in cycling cells, accentuating a subtle change in targeting patterns of EB1 between interphase and mitosis. Interestingly, uniform binding of EB1 along the entire microtubule was observed in tissue culture cells after taxol treatment and on nocodazoleresistant microtubules in interphase cells (Tirnauer and Mitchison, unpublished data). Consistent with the ability to bind the microtubule wall independent of polymerization status, EB1 displayed a weak, micromolar binding affinity for purified microtubules in our copelleting assay.

\section{EB1 Binds to Polymerizing Microtubule Plus Ends and along the Microtubule Wall, Coupled to Rapid Dissociation Throughout}

In forming a comet shape, EB1 behaves similarly to the other microtubule plus end tracking proteins, or + tips (Schuyler and Pellman, 2001a). From our localization and FRAP experiments, it seems that EB1 binds to microtubules by two mechanisms, one specific to polymerizing plus ends and one occurring along the length of the microtubule wall. Possible mechanisms for enhanced binding to polymerizing microtubule ends include copolymerization with tubulin, transport of EB1 to the microtubule end, or enhanced binding to a structural (such as the unrolled sheet) or chemical (such as the GTP cap) property of the microtubule end (horizontal arrows in Figure 5). Our speckling data showing that EB1 remained static with respect to the comets rules out the possibility that EB1 is transported along the microtubule. Although our data do not distinguish among the other possibilities, we saw very little tubulin monomer in our EB1 immunoprecipitations. Thus, we currently favor the idea that EB1 recognizes a property of polymerizing microtubule ends. The best characterized protein with regard to microtubule plus end binding, CLIP-170, is thought to copolymerize with tubulin, based on binding to tubulin dimers and localization patterns (Diamantopoulos et al., 1999). Because EB1 is $\sim 1 / 100$ of the tubulin concentration in egg extracts, 
EB1 may generate comets by a different mechanism from CLIP-170.

Regarding the binding to microtubule walls, straightforward comparisons of our FRAP data and relative brightness analysis indicate that the on rate of EB1 for microtubules and the number of EB1 binding sites is relatively constant during interphase and CSF arrest (Figure 5, vertical arrows). The fraction of bound sites, in contrast, seems to be less during CSF arrest, and this is likely due to the threefold faster dissociation rate. The slower dissociation rate of EB1 from interphase microtubules predicts the higher steady-state fluorescence intensity, representative of EB1 concentration, on these microtubules. EB1 shares slower dissociation during interphase with several other microtubule-binding proteins (Bulinski et al., 2001).

Because at one point in time, every region of the microtubule was transiently the plus end, generation of the comet shape requires not only enhanced binding to the end, but also continuous dissociation distally. Our fluorescence recovery analysis of microtubule wall binding, compared with fluorescence decay over time analysis of EB1 in the comet, give similar half-lives of dissociation on the order of a few seconds. It is therefore likely that the mechanism of EB1 dissociation is similar along the entire length of the microtubule, including the end (Figure 5, vertical arrows). Similar dissociation rates from the microtubule wall and the end predict that the length of the comet should correlate with the polymerization rate. We found a moderate correlation between polymerization rate and comet size, but the best fit to a first order dissociation curve was found when we analyzed the decay of fluorescence at a single point in the comet over time. This result is somewhat similar to the exponential decay seen in actin comet tails, where the net actin polymerization rate is constant and exponential decay is superimposable in space and time (Theriot et al., 1992). In EB1 comets, the additional phenomena of variable polymerization rates and microexcursions, as well as rebinding to intact microtubules, created a more complex situation where the temporal decay at a single point produced a simple exponential, but the spatial distribution at a single time revealed these additional phenomena. The faster dissociation during mitosis remodels EB1 into comets at microtubule ends, an ideal pattern for microtubule end-specific recognition by other proteins in mitotic cells such as kinetochores and polarity determinants.

\section{ACKNOWLEDGMENTS}

We thank Paul Maddox for assistance with the photobleaching experiments and Michelle Shirasu-Hiza for helpful comments on the manuscript. This study was supported by National Institutes of Health grants K08 DK-02578 (to J.S.T.), GM-24364 (to E.D.S.), and GM-39565 (to T.J.M.).

\section{REFERENCES}

Adames, N.R., and Cooper, J.A. (2000). Microtubule interactions with the cell cortex causing nuclear movements in Saccharomyces cerevisiae. J. Cell Biol. 149, 863-874.

Akhmanova, A., et al. (2001). Clasps are CLIP-115 and -170 associating proteins involved in the regional regulation of microtubule dynamics in motile fibroblasts. Cell 104, 923-935.
Arnal, I., Karsenti, E., and Hyman, A.A. (2000). Structural transitions at microtubule ends correlate with their dynamic properties in Xenopus egg extracts. J. Cell Biol. 149, 767-774.

Beinhauer, J.D., Hagan, I.M., Hegemann, J.H., and Fleig, U. (1997). Mal3, the fission yeast homolog of the human APC-interacting protein EB-1, is required for microtubule integrity and the maintenance of cell form. J. Cell Biol. 139, 717-728.

Berrueta, L., Kraeft, S.-K., Tirnauer, J.S., Schuyler, S., Chen, L.B., Hill, D.E., Pellman, D., and Bierer, B. (1998). The adenomatous polyposis coli-binding protein EB1 is associated with cytoplasmic and spindle microtubules. Proc. Natl. Acad. Sci. USA 95, 1059610601.

Bu, W., and Su, L.K. (2001). Regulation of microtubule assembly by human EB1 family proteins. Oncogene 20, 3185-3192.

Bulinski, J.C., Odde, D.J., Howell, B.J., Salmon, T.D., and WatermanStorer, C.M. (2001). Rapid dynamics of the microtubule binding of ensconsin in vivo. J. Cell Sci. 114, 3885-3897.

Cassimeris, L., and Spittle, C. (2001). Regulation of microtubuleassociated proteins. Int. Rev. Cytol. 210, 163-226.

Davis, A., Sage, C.R., Wilson, L., and Farrell, K. (1993). Purification and biochemical characterization of tubulin from the budding yeast Saccaromyces cerevisiae. Biochemistry 32, 8823-8835.

Desai, A., Maddox, P.S., Mitchison, T.J., and Salmon, E.D. (1998). Anaphase A chromosome movement and poleward spindle microtubule flux occur At similar rates in Xenopus extract spindles. J. Cell Biol. 141, 703-713.

Desai, A., and Mitchison, T.J. (1997). Microtubule polymerization dynamics. Annu. Rev. Cell Dev. Biol. 13, 83-117.

Desai, A., Murray, A.W., Mitchison, T.J., and Walczak, C.E. (1999a). The use of Xenopus egg extracts to study mitotic spindle assembly and function in vitro. Methods Cell Biol. 61, 385-412.

Desai, A., Verma, S., Mitchison, T.J., and Walczak, C. (1999b). Kin I kinesins are microtubule destabilizing enzymes. Cell 96, 69-78.

Diamantopoulos, G.S., Perez, F., Goodson, H.V., Batelier, G., Melki, R., Kreis, T.E., and Rickard, J.E. (1999). Dynamic localization of CLIP-170 to microtubule plus ends is coupled to microtubule assembly. J. Cell Biol. 144, 99-112.

Fodde, R., et al. (2001). Mutations in the APC tumor suppressor gene cause chromosomal instability. Nat. Cell Biology 3, 433-438.

Gard, D.L., and Kirschner, M.W. (1987). Microtubule assembly in cytoplasmic extracts of Xenopus oocytes and eggs. J. Cell Biol. 105, 2191-2201.

Grego, S., Cantillana, V., and Salmon, E.D. (2001). Microtubule treadmilling in vitro investigated by fluorescence speckle and confocal microscopy. Biophys. J. 81, 66-78.

Hyman, A., Drechsel, D., Kellogg, D., Salser, S., Sawin, K., Steffen, P., Wordeman, L., and Mitchison, T. (1991). Preparation of modified tubulins. Methods Enzymol. 196, 478-485.

Hyman, A.A., Salser, S., Drechsel, D., Unwin, N., and Mitchison, T.J. (1992). Role of GTP hydrolysis in microtubule dynamics: information from a slowly hydrolysable analogue, GMPCPP. Mol. Biol. Cell 3, 1155-1167.

Janosi, I.M., Chretien, D., and Flyvbjerg, H. (1998). Modeling elastic properties of microtubule tips and walls. Eur. Biophys. J. 27, 501513.

Kaplan, K.B., Burds, A.A., Swedlow, J.R., Bekir, S.S., Sorger, P.K., and Nathke, I.S. (2001). A role for the adenomatous polyposis coli protein in chromosome segregation. Nat. Cell Biol. 3, 429-432.

Kinoshita, K., Arnal, I., Desai, A., Drechsel, D.N., and Hyman, A.A. (2001). Reconstitution of physiological microtubule dynamics using purified components. Science 294, 1340-1343. 
Korinek, W.S., Copeland, M.J., Chaudhuri, A., and Chant, J. (2000). Molecular linkage underlying microtubule orientation toward cortical sites in yeast. Science 287, 2257-2259.

Lee, L., Klee, S.K., Evangelista, M., Boone, C., and Pellman, D. (1999). Control of mitotic spindle position by the Saccharomyces cerevisiae formin Bni1p. J. Cell Biol. 144, 947-961.

Maddox, P.S., Bloom, K.S., and Salmon, E.D. (2000). The polarity and dynamics of microtubule assembly in the budding yeast Saccharomyces cerevisiae. Nat. Cell Biol. 2, 36-41.

Mandelkow, E.M., Mandelkow, E., and Milligan, R. (1991). Microtubule dynamics and microtubule caps: a time-resolved cryo-electron microscopy study. J. Cell Biol. 114, 977-991.

Miller, R.K., Cheng, S.-C., and Rose, M.D. (2000). Bim1p/Yeb1p mediates the Kar9p-dependent cortical attachment of cytoplasmic microtubules. Mol. Biol. Cell 11, 2949-2959.

Mimori-Kiyosue, Y., Shiina, N., and Tsukita, S. (2000). The dynamic behavior of the APC-binding protein EB1 on the distal ends of microtubules. Curr. Biol. 10, 865-868.

Mitchison, T.J., and Kirschner, M.W. (1986). Isolation of mammalian centrosomes. Methods Enzymol. 134, 261-268.

Nakamura, M., Zhou, X.Z., and Lu, K.P. (2001). Critical role for the EB1 and APC interaction in the regulation of microtubule polymerization. Curr. Biol. 11, 1062-1067.

Olmsted, J.B., Stemple, D.L., Saxton, W.M., Neighbors, B.W., and McIntosh, J.R. (1989). Cell cycle-dependent changes in the dynamics of MAP 2 and MAP 4 in cultured cells. J. Cell Biol. 109, 211-223.

Palazzo, A.F., Cook, T.A., Alberts, A.S., and Gundersen, G.G. (2001). mDia mediates Rho-regulated formation and orientation of stable microtubules. Nat. Cell Biol. 3, 723-729.

Parsons, S.F., and Salmon, E.D. (1997). Microtubule assembly in clarified Xenopus egg extracts. Cell Motil. Cytoskeleton 36, 1-11.

Rusan, N.M., Fagerstrom, C.J., Yvon, A.-M.C., and Wadsworth, P. (2001). Cell cycle-dependent changes in microtubule dynamics in living cells expressing green fluorescent protein- $\alpha$ tubulin. Mol. Biol. Cell 12, 971-980.
Schroer, T.A. (2001). Microtubules don and doff their caps: dynamic attachments at plus and minus ends. Curr. Opin. Cell Biol. 13, 92-96.

Schuyler, S., and Pellman, D. (2001a). Microtubule "plus-end-tracking proteins": the end is just the beginning. Cell 105, 421-424.

Schuyler, S.C., and Pellman, D. (2001b). Search, capture and signal: games microtubules and centrosomes play. J. Cell Sci. 114, 247-255.

Theriot, J.A., Mitchison, T.J., Tilney, L.G., and Portney, D.A. (1992). The rate of actin-based motility of intracellular Listeria monocytogenes equals the rate of actin polymerization. Nature 357, 257-260.

Tirnauer, J.S., and Bierer, B.E. (2000). EB1 proteins regulate microtubule dynamics, cell polarity, and chromosome stability. J. Cell Biol. 149, 761-766.

Tirnauer, J.S., Canman, J.C., Salmon, E.D., and Mitchison, T.J. (2002). EB1 targets to kinetochores with attached polymerizing microtubules. Mol. Biol. Cell 13, (in press).

Tirnauer, J.S., O'Toole, E., Berrueta, L., Bierer, B., and Pellman, D. (1999). Yeast Bim1p promotes the G1-specific dynamics of microtubules. J. Cell Biol. 145, 993-1007.

Tran, P.T., Joshi, P., and Salmon, E.D. (1997). How tubulin subunits are lost from the shortening ends of microtubules. J. Struct. Biol. 118, 107-118.

Wadsworth, P. (1999). Regional regulation of microtubule dynamics in polarized, motile cells. Cell Motil. Cytoskeleton 42, 48-59.

Walker, R.A., et. al. (1988). Dynamic instability of individual microtubules analyzed by video light microscopy: rate constants and transition frequencies. J. Cell Biol. 107, 1437-1448.

Waterman-Storer, C.M., Desai, A., Bulinski, J.C., and Salmon, E.D. (1998). Fluorescent speckle microscopy, a method to visualize the dynamics of protein assemblies in living cells. Curr. Biol. 8, 12271230 .

Waterman-Storer, C.M., and Salmon, E.D. (1999). Fluorescent speckle microscopy of microtubules: how low can you go? FASEB J. 13, S225-S230.

Zhai, Y., Kronebusch, P.J., and Borisy, G.G. (1995). Kinetochore microtubule dynamics at the metaphase-anaphase transition. J. Cell Biol. 131, 721-734 